International Journal of Biomedicine | June 2019 - Volume 9, Issue Suppl_1: Abstracts From the Second Russian International Conference "Cryo-electron microscopy 2019: achievements and prospects"

ORAL ABSTRACT PRESENTATIONS

SESSION TITLE: APPLICATIONS OF CRYO-EM IN MEDICINE

DOI: 10.21103/IJBM.9.Suppl_1.OR14

\title{
Abstract OR-14: Fibril Structure and Interface Polymorphism of Amyloid-B(1-42)
}

\author{
Gunnar F. Schröder \\ Forschungszentrum Jülich, Jülich, Germany
}

Background: Amyloids are associated with many diseases. The recent breakthrough in the cryoEM technique has made it possible to study to structure of amyloid fibrils by cryo-EM to high resolution. The technique will be introduced and applications to different amyloid structures presented, in particular the structure of the amyloid- $\beta$ fibril (A $\beta 1-42)$, which is involved in Alzheimer's disease. Furthermore, new image processing methods will be presented to reconstruct macromolecular motions from singleparticle image data.

Methods: Single-particle cryo-EM was used to determine helical reconstructions of different fibrillar structures. Furthermore, an approach is presented to determine the principal motions of macromolecules from single-particle data.

Typical classification methods aim for maximizing density differences between different models. However, density variance is not the same as conformational variance (i.e. variance of atomic model coordinates), and for interpreting molecular motions, conformational variance is most relevant. We present an approach to determine this conformational variance.

Results: The structure of an amyloid- $\beta$ fibril (A $\beta 1-42)$ and polymorphs of it were determined. In addition, the structure of an SH3 amyloid fibril is presented, which has been extensively studied as a model system for amyloid formation.

Conclusion: Cryo-EM reveals high-resolution structural details of amyloid fibrils.

Key Words: amyloid fibrils • helical reconstruction • Alzheimer disease

International Journal of Biomedicine. 2019;9 Suppl 1: S11. doi: 10.21103/IJBM.9.Suppl_1.OR14

C2019 International Medical Research and Development Corporation 